\title{
Rethinking grant review
}

\begin{abstract}
The US government is re-examining how it funds science, but it remains to be seen whether the administration can overcome tradition and bureaucratic inertia and achieve a substantial renovation of the grants peer-review system.
\end{abstract}

T he US National Institutes of Health (NIH) has embarked on a comprehensive overhaul of its grant review process. Director Elias Zerhouni challenged members of the NIH and the scientific community to find a way to "fund the best science, by the best scientists, with the least administrative burden." The NIH has made a tremendous effort to obtain input from across the scientific community on how these goals might be accomplished. The process has included a request for information that elicited over 2,600 responses, regional meetings with stakeholders and consultation with professional societies.

The current peer-review system is clearly overburdened by limited finances and increasing numbers of applications. The 2008 US federal budget includes less than a $1 \%$ increase in NIH funding, which is actually a decrease in the money available to support scientific research when adjusted for inflation. Although this news is not surprising in the current political climate, it is certainly disheartening for an institution that has seen little significant budget growth since 2003. Even more troubling is that the overall grant funding rate has dropped substantially. About $30 \%$ of R01 proposals were funded in 2003, but only $20 \%$ were funded in 2006 , a change due almost entirely to a major increase in the number of proposals submitted.

More proposals require more reviewers, and the tight funding situation makes it difficult for researchers to commit time to serving on grant review committees rather than increasing the productivity of their own laboratories. Scientific review officers, who administer the peer-review process at $\mathrm{NIH}$, report having difficulty recruiting reviewers, particularly high-caliber, established investigators. Another problem with the current system is that it selects against risky projects, particularly in times of limited resources. Pilot data, once considered a feature of the strongest proposals, are now required just to be competitive, and the feasibility of the proposed research is a major concern for reviewers. With so little money, it might seem to make sense to support the research that is most likely to produce results, but under this philosophy, the successful proposals tend to be the most conservative, which typically produce only incremental advances in knowledge.

Some of the ideas under consideration to address these problems deserve to be called revolutionary. One possibility would be to shorten grant proposals from 25 to as few as 7 pages, shifting the focus from approach and preliminary results to innovation and potential impact. Another proposal, changing the scoring system, would shift the focus of the review process, forcing reviewers to consider innovation, impact and the individual track record of the investigator, rather than the methodology and feasibility of the particular study proposed. Also under consideration is a review board model, in which proposals would be sent to specialized reviewers for evaluation of technical issues and then to another set of reviewers who would look at both the specialized reports and the big picture.

Other suggestions tend more in the direction of business as usual. For example, to improve review quality, the NIH could train its reviewers, provide incentives to attract and retain the best reviewers, rate reviewers, identify reviewers or place an ombudsperson on each study section. Though this class of solutions would result in comparatively minor changes in the way grants are reviewed, they could be implemented quickly and without extensive effort.

Two committees have been charged with deciding which proposals to recommend to Zerhouni and his advisory committee. One is an internal working group composed of $\mathrm{NIH}$ directors, and the other is an external working group composed of NIH-funded researchers. In February, they will present a 'white paper' outlining the areas they believe to be most in need of change and how those changes might be implemented. On the basis of these recommendations, Zerhouni and his advisory committee will decide how to proceed.

Some of the more radical proposals may need to be tested in small trials before a sensible decision can be made about their value. Once the director decides which ones to pursue, the projects will be assigned to the appropriate centers in the NIH. These centers will then be responsible for designing methods to evaluate the effects of the new approaches. This process is intended to begin as soon as March.

Institutional inertia may make it difficult to obtain approval for the more serious changes, but there is reason to believe that this effort has support from the top of NIH. The directors of many of the institutes have actively participated in the endeavor, and both Zerhouni and Raynard Kington, Principal Deputy Director of the $\mathrm{NIH}$, have been highly engaged in re-examining how the institute funds research. These are hopeful signs that the NIH is committed to changing the way things work, but evaluating and implementing significant changes will require substantially more effort.

One serious shortcoming of the process is that no financial support has been specifically earmarked yet for realization of these new ideas. Those assigned responsibility for the implementation of individual projects will be able to apply for support from the general pool of funds available for evaluation of NIH-run programs, but such funds require proposal submission and are not guaranteed. In addition, some projects may be funded through redistribution of money already budgeted to the institute where the pilot is being managed. If the NIH is serious about revamping its peer review system, a project that is long overdue, then it will need to provide enough resources to see the process through to the end. 